\title{
On Incentives in Global Wireless Communities
}

\author{
Gergely Biczók, László Toka, Attila Vidács, and Tuan A. Trinh \\ High Speed Networks Lab \\ Dept. of Telecommunications and Media Informatics \\ Budapest University of Technology and Economics \\ \{biczok, toka, vidacs, trinh\}@tmit.bme.hu
}

\begin{abstract}
The wireless community networking paradigm shows great promise in achieving a global status. However, both user participation and support from traditional Internet Service Providers (ISPs) play key roles in creating worldwide coverage; for this end a viable incentive system is essential. In this paper we study the economic interactions between users, ISPs and community providers. Our main contribution is threefold. First, we propose a model of the global wireless community concept as a Stackelberg game of two levels and construct the respective payoff functions of each player. Second, we show how both users and ISPs may fail to join the community in equilibrium. Third, we explore the parameter space of the mechanism designer and show how the technology diffusion process and expected payoffs can be controlled by adjusting roaming prices and revenue shares.
\end{abstract}

\section{Categories and Subject Descriptors}

C.2.1 [Network Architecture and Design]: Wireless communication; C.2.3 [Computer-Communication Networks]: Network Operations

\section{General Terms}

Economics, Management, Theory

\section{Keywords}

global wireless community networks, incentives, network economics, game theory

\section{INTRODUCTION}

User-provided networking has seen its stock rising significantly. While some see this concept as an interesting but only moderately viable alternative to the traditional ISP-centric model, others believe it has the potential to induce a complete shift in Internet communication patterns and form a foundation for the future wireless Internet. A working prototype of such a global wireless community network already exists: the FON WiFi system [1]. Community

Permission to make digital or hard copies of all or part of this work for personal or classroom use is granted without fee provided that copies are not made or distributed for profit or commercial advantage and that copies bear this notice and the full citation on the first page. To copy otherwise, to republish, to post on servers or to redistribute to lists, requires prior specific permission and/or a fee.

U-NET'09, December 1, 2009, Rome, Italy.

Copyright 2009 ACM 978-1-60558-750-9/09/12 ...\$10.00. members, referred to as Foneros, share their home Internet connections and gain access to free WiFi at other locations. There are three different types of users: Linus, Bill, and Alien. A Linus has a "La Fonera" WiFi router, shares WiFi and gets free roaming at any FON Spot. A Bill, having the same rights as a Linus, gets further $50 \%$ of the revenues when a visitor buys a FON pass at her FON Spot. An Alien does not share an Internet connection, nevertheless she accesses FON Spots by purchasing short-term passes. Since its inception in 2005, a number of prolific companies have partnered the FON movement: Google, Skype and British Telecom among others. Furthermore, FON claims to have more than 300,000 sharingenabled routers and more than a million users all over the world. We argue that although FON and other (global) wireless community networking frameworks (Whisher [2], WeFi [3] and local Meraki [4] among others) show great promise, their ultimate success depends on properly designed incentive mechanisms which facilitate both the participation of users and the cooperation of ISPs. Currently the majority of ISPs do not allow connection sharing for their subscribers, which is the most significant obstacle in the way of creating a global wireless community.

There is some existing literature on pricing and participation in wireless community networks. A charging model for wireless social community networks without a centralized authority is proposed by authors of [5]. In [6] and [7] authors study the issue of modeling user subscription, mobility and coverage evolution using both analytical and simulation approaches with the objective of finding optimal subscription fees. Furthermore, the co-existence of WAN and WiFi technologies and cooperation between the respective providers is studied in [8]. Unlike the existing literature that focuses either on local community networks or provider-based WiFi, our work aims at modeling a global wireless community, where ISPs also play an important role, and the presence of incentives and the proper dynamics of deployment are crucial. As evidenced by the growing number of ISPs partnering with FON (BT, Neuf, Time Warner, etc.), studying the economic interactions among users, ISPs and community providers is a key topic. To the best of our knowledge this work is the first to explore this issue.

In this paper we investigate the economic interactions in global wireless user-provided networks with regard to users, ISPs and community providers (called mediators) through a simple analytic model. The main contribution of this paper is threefold. First, we develop a model of the global wireless community concept as a Stackelberg game [9] of participation on two levels (the mediator acting as a mechanism designer in the leader role and the users and ISPs as followers) and construct the respective payoff functions of the mediator-, user- and ISP-type players (Sec. 2). Second, we analyze equilibrium properties of the game for users and ISPs, and show that users and ISPs refuse to join the community network un- 
der specific circumstances (Sec. 3.1). Third, we explore the role of the mechanism designer (the mediator) and show how the expected payoffs and the technology diffusion process are affected by tunable parameters (Sec. 3.2). Specifically, setting the roaming and entry costs properly, the mediator can side either with the users or the ISPs by realizing its maximum profit close to their respective maxima. Moreover, dynamically adjusting revenue shares can help in overcoming the problem of stagnating technology diffusion. We also outline possible future research directions in Sec. 4.

\section{SYSTEM MODEL}

We model the economic interactions of a global wireless community network as a two-level game. We introduce the notion of a mediator (community provider) which operates as a mechanism designer in the role of the leader of our Stackelberg (leader-follower) game. At the first level, the mediator decides on the distribution of money flows among the other participants (followers), i.e., users and ISPs. At the second level, Internet subscribers and providers play a one-shot game where the users' possible strategies consist of the participation decision, i.e., one may join the Internet connection sharing community or stay out and pay when roaming. Moreover, the ISPs decide whether to support end-users' access sharing, i.e., to opt in for the user-provided networking community or not.

In this section we make some ground assumptions in order to define instantaneous (for comparability reasons) payoff functions for each type of player. In Sec. 3.1 we analyze the game that arises on these utilities and costs and we present our findings on the equilibrium states.

\subsection{Assumptions}

Here we list our assumptions which enable us to build a tractable model.

\section{ASSUMPTION 1. (Simplifications for modeling)}

- the user and ISP populations are static, thus we can model the system as two one-shot games at the second level of the Stackelberg game;

- every user has broadband Internet access at home and electricity is relatively cheap, hence community members' shared WiFi networks are only used by roaming users and their Internet access boxes and wireless routers are never turned off;

- users' home Internet connections have excess capacity, so sharing it causes only negligible reduction in quality of experience for the home user (also, traffic prioritization is applied at the home router [1]);

- the mediator-tuned price provides the cheapest option among roaming WiFi networks, so users favor community wireless networks to other commercial WiFi hot-spots, if available;

- users may become community members regardless of their ISPs' decisions (however we consider lower average member rate in the subscriber base of a non-supportive ISP in Sec. 2.4), and they do not switch ISPs. Furthermore, we do not target broadband access pricing, therefore we simply assume that ISPs have similar subscriber numbers and that they do not lose existing and/or potential subscribers due to their policy on connection sharing.

\subsection{The mediator}

In the following we define the role of the mediator as the leader in our game through her payoff function. Exact values of parameters $\alpha, \beta, c_{o}$ and $c_{i}$ (see below) are determined by this entity.
DEFINITION 1. (Mediator payoff) The instantaneous payoff function $\Pi_{m}$ of the mediator is defined as

$$
\Pi_{m}=G T n_{o} c_{o}(1-\alpha-\beta)-c_{m}+n_{i}\left(c_{i}-c_{i}^{\prime}\right)
$$

where

- $G \in[0 . .1]$ represents a user's mobility and demand to be online when roaming, i.e., the probability of the given user's appearance at a given geographic location and requesting Internet connection;

- $T \in[0 . .1]$ represents the technology penetration, i.e., the ratio of insiders within the user population, namely the probability that at least one community member provides Internet access WiFi at a given geographic location;

- $n_{o}$ is the global number of outsiders, i.e., users who do not share their broadband access;

- $n_{i}$ is the global number of insiders, i.e., users who share their broadband access $\left(n_{u}=n_{o}+n_{i}\right.$ is the global number of users);

- $\alpha \in[0 . .1]$ is the ratio of the roaming fee that the insider receives from an outsider's payment $\left(c_{o}\right)$ through the mediator when she uses the insider's connection;

- $\beta \in[0 . .1]$ is the ratio of the roaming fee that the ISP receives from an outsider's payment $\left(c_{o}\right)$ through the mediator when she uses the shared Internet connection of one of the ISP's subscribers $(\alpha+\beta<1)$;

- $c_{o}$ is the time-unit price for roaming WiFi access, paid by the outsider;

- $c_{i}$ represents the insider's entry cost (e.g., the price of a system-dedicated box, that is supposed to be upgraded periodically) paid to the mediator, discounted to time-unit;

- $c_{m}$ represents the time-unit operational cost of the mediator;

- $c_{i}^{\prime}$ denote the manufacturing cost of a single sharing-enabled home access router and accessories, discount-ed to timeunit.

\subsection{The user}

Next, we define the payoff function for the two possible user strategies, i.e., joining the global community (insider) or staying out (outsider).

DEFINITION 2. (User payoff) The instantaneous payoff function $\Pi_{o}$ (resp. $\Pi_{i}$ ) of an outsider (resp. insider) is defined as

$$
\begin{gathered}
\Pi_{o}=G T\left(u_{o}-c_{o}\right) \\
\Pi_{i}=G T\left(u_{i}+R n_{o} c_{o} \alpha \frac{1}{T^{2}}\right)-c_{i}
\end{gathered}
$$

where

- $u_{o}$ is an outsider's time-unit utility for being online;

- $u_{i}$ is an insider's time-unit utility for being online;

- $R \in[0 . .1]$ denotes the geographic relevance of the insider's home, i.e., the fraction of the roaming outsiders that appear in the range of her wireless router's coverage at a given moment; 
- the multiplicative term $\frac{1}{T^{2}}$ reflects the competition among the insiders at a given geographic location, i.e., when the membership ratio grows in the user population, the number of competing insiders at a given location is assumed to grow quadratically, therefore every insider's income generated by outsiders drops at the same rate.

\subsection{The Internet Service Provider}

We model the decision possibilities of the ISPs with the following game-theoretic strategies. We call an ISP a defector, if she does not support the community sharing of Internet access, and an adopter if she does. Furthermore, we assume that a fraction of a defector ISP's subscribers will not become insiders despite their intention to do so, due to the risk of violating their broadband contracts.

DEFINITION 3. (ISP payoff) The instantaneous payoff function $\Pi_{d}$ (resp. $\Pi_{a}$ ) of a defector (resp. adopter) ISP is defined as

$$
\begin{gathered}
\Pi_{d}=G(T-\gamma) n_{o}\left(-c_{t}\right) \\
\Pi_{a}=G(T+\gamma) n_{o}\left(c_{o} \beta-c_{t}\right)-c_{a}
\end{gathered}
$$

where

- $\gamma$ is the obedience parameter, i.e., $2 \gamma$ represents the difference between the ratio of insiders at an adopter ISP versus a defector ISP (remember that users can risk becoming insiders even if their ISP does not allow it);

- $c_{t}$ represents the time-unit cost associated with carrying extra traffic caused by outsiders using the shared connections at the ISP's subscribers who became insiders;

- $c_{a}$ is the ISP's time-unit cost of adoption of the community sharing concept, incorporating trust, security and infrastructural expenditures.

\section{ANALYSIS}

In this section we investigate the characteristics of the game that we defined previously. Building upon the presented payoffs, and the assumption that every participant behaves selfishly (in the gametheoretic sense), first we deduce the expected number of insiders in the system, then we analyze the effects of different parameters on this latter. We follow our homogeneous case assumptions:

AsSUMPTION 2. (Homogeneous case)

- $G=G_{o}=G_{i}$, i.e., mobility and connectivity demand among users are homogeneous

- $u_{o}=u_{i}$, the utility of being online is the same for insiders and outsiders,

- $T=\frac{n_{i}}{n_{u}}$, i.e., insiders are evenly distributed geographically;

- $R$ is also homogeneous, i.e., every insider's home router is placed at an evenly visited location,

- ISPs share subscribers evenly (excluding $\gamma$ ). Note that weighing ISP payoffs with their user base is omitted (i.e., they do not compete for users), hence user, ISP and mediator payoffs cannot be compared directly.

Although in (1) we provided a heuristic payoff function for the mediator reflecting her income, in this paper we do not target the analysis of her actual goals that can be various, e.g., maximization of social welfare or individual profit, therefore we do not address the issue of her best response strategy (we do provide some numerical insight in Sec. 3.2) [9]. We suppose that the parameters constituting her strategy are determined steadily so the followers (i.e., users and ISPs) could choose their strategies based upon those. Hence, our equilibrium analysis of the followers' game is provided in this perspective.

\subsection{Critical mass}

User game. Selfish users join the sharing community and become insiders if their payoffs get higher than the payoffs they get as outsiders, i.e.,

$$
\Pi_{i}>\Pi_{o} .
$$

We model the game among users by a simple one-shot game, and we derive the stable outcomes, i.e., the pure and mixed strategy Nash equilibria [9] (where no player gains benefit by unilaterally changing her strategy) based on the relation between the payoff function and the number of insiders. By substituting (2) and (3) into (6) we get the following constraint:

$$
c_{i}<c_{o} G\left(T+\frac{R}{T} \alpha n_{o}\right) .
$$

Using our assumption on homogeneous technology penetration and simplifying the expression, we get:

$$
n_{i}^{2} \frac{1}{n_{u}}-n_{i}\left(\frac{c_{i}}{c_{o} G}+n_{u} R \alpha\right)+n_{u}^{2} R \alpha>0 .
$$

Solving the quadratic for $n_{i}$ we infer roots $n_{i_{1,2}}^{u *}$, such as $n_{i}<n_{i_{1}}^{u *}$ or $n_{i}>n_{i_{2}}^{u *}$ satisfies (8). Stability, from the users' perspective, is highly affected by the number of insiders.

\section{PROPOSITION 1. (Equilibria of the user game)}

1. Every user playing the insider strategy is a pure strategy Nash equilibrium, if (8) has at most one real root or it has two real roots, but $n_{i_{1}}^{u *}>n_{u}$. In this case an insider's payoff is higher than an outsider's, irrespective of the actual number of insiders $n_{i}$.

2. Every user playing the mixed strategy $P($ insider $)=\frac{n_{i_{1}}^{u *}}{n_{u}}$ and $P($ outsider $)=1-P($ insider $)$ is a mixed strategy Nash equilibrium, if (8) has two real roots and $n_{i_{1}}^{u *} \leq n_{u}\left(n_{i_{1}}^{u *}>0\right.$ always holds). In this case $\left(n_{i}=n_{i_{1}}^{u *}\right)$ no outsider would become insider to have a negative payoff growth and similarly no insider would leave the community since the outsider payoff gets worse as the number of insiders shrinks at this point (see (2)).

3. There are two Nash equilibria at $n_{i}=n_{i_{1}}^{u *}$ (mixed, see previous) and $n_{i}=n_{u}$ (pure, every user is insider), if both real roots exist and $n_{i_{2}}^{u *}<n_{u}$.

Proposition 1 shows an important characteristic of the system: under certain circumstances, the insider population can stop growing. This phenomenon hinders technology diffusion and it can impact future payoffs in a negative way. See Sec. 3.2 for a practical solution to this problem.

ISP game. Similarly to users, when an ISP opts in for the community Internet access sharing, it expects higher revenues by doing so:

$$
\Pi_{a}>\Pi_{d}
$$




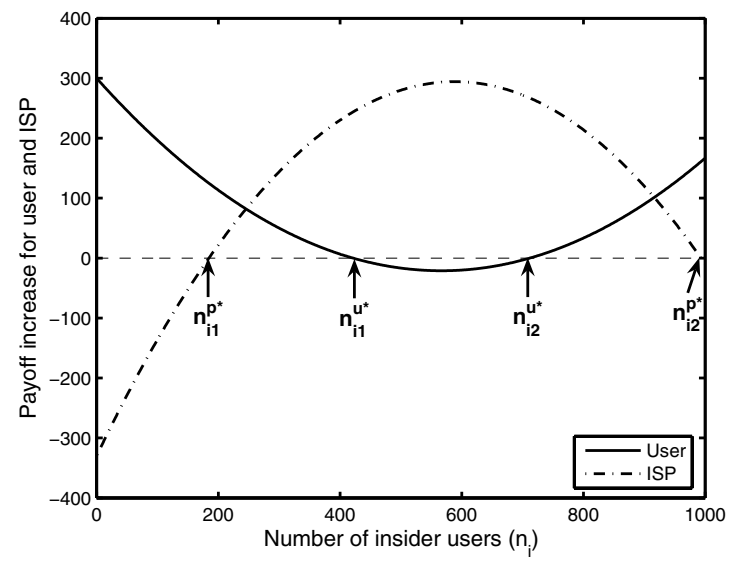

Figure 1: Expected revenue for user and ISP

By substituting (4) and assuming homogeneous technology penetration $\left(T=\frac{n_{i}}{n_{u}}\right)$ we get the following constraint:

$$
G\left(n_{u}-n_{i}\right)\left(c_{o} \beta\left(\frac{n_{i}}{n_{u}}+\gamma\right)-2 \gamma c_{t}\right)-c_{a}>0 .
$$

Solving the quadratic for $n_{i}$ we get roots $n_{i_{1,2}}^{p *}$, such as $n_{i_{1}}^{p *}<$ $n_{i}<n_{i_{2}}^{p *}$ satisfies (10). Since in our simple model the ISPs' strategies do not affect the users' or even each other's decisions, in Nash equilibrium an ISP's best response strategy is based only on the stability characteristics of the game among the users, i.e., the number of insiders present.

\section{Proposition 2. (Equilibria of the ISP game)}

1. Every ISP adopting is a pure strategy Nash equilibrium, if $n_{i_{1}}^{p *}<n_{i}<n_{i_{2}}^{p *}$.

2. Every ISP defecting is a pure strategy Nash equilibrium, if (10) has at most one real root; or there are two real roots such as $n_{i}<n_{i_{1}}^{p *}$ or $n_{i}>n_{i_{2}}^{p *}$; or irrespective of the roots, if $n_{i}=n_{u}$

Thus, ISPs are set to join the community when there is already a substantial insider base, but there is still a sufficient number of outsiders. This can cause problems at low technology penetration. See how dynamically adjusting revenue shares can be a potential solution to this problem in Sec. 3.2.

Proofs of Propositions 1 and 2 can be derived in a straightforward manner, thus we omit them. For the sake of understanding, results are visualized in Figure 1. Here, the respective payoff differences for both the user $\left(\Pi_{i}-\Pi_{o}\right)$ and the ISP $\left(\Pi_{a}-\Pi_{d}\right)$ are plotted against the number of insiders $\left(n_{i}\right)$ for a fixed parameter set. Note that in this setting two real roots exist for both (8) and (10) such as $n_{i_{1,2}}^{u *}<n_{u}$ and $n_{i_{1,2}}^{p *}<n_{u}$.

\subsection{Exploring the parameter space}

The success of the technology, materializing in more users and ISPs joining, depends significantly on the exact setting of corresponding fees and other parameters. Therefore, we hereby explore the design space by tweaking a single parameter (except for revenue shares, where we fix $\alpha+\beta$ ) and fixing all others at a time (see Figure 2). Note that setting roaming $\operatorname{cost} c_{o}$, entry cost $c_{i}$, and revenue shares $\alpha$ and $\beta$ is in the power of the mediator. We have to emphasize that although we have to assign exact values to parameters to be able to evaluate their impact, this evaluation should be considered as more qualitative than quantitative. Our goal is to draw attention to some basic economic implications of the current business model of user-provided networking. In the following, for practical reasons we consider $n_{u}=1000$ as the population size.

Adoption cost $\left(c_{a}\right.$, Figure 2(a)). Being a simple additive term in the ISP's payoff function, increasing adoption cost results in diminishing profits. It can be observed that even at relatively low $c_{a}$, the number of insiders should be reasonably high in the user population (critical mass) for the ISPs to be worth joining. Also, if $c_{a}$ is high enough, it may not be worth joining if technology penetration is too high (late market entry, $c_{a}=200, T>0.85$ ).

Traffic-dependent cost $\left(c_{t}\right.$, Figure 2(b)). When $c_{t}$ is relatively low, an ISP has every incentive to join with her maximum payoff realized around $T=0.5$, i.e., when the number of outsiders and insiders are about even. If we increase the traffic dependent cost (remember, this traffic is coming from outsiders using the shared broadband in the ISP's network at her insiders) network providers need higher technology penetration to make profit from joining. Close to full penetration this profit vanishes, since there is no outsider who pays for roaming.

User obedience ( $\gamma$, Figure 2(c)). The larger is the ratio of users that choose to be insider even if their ISP does not allow it, the less insiders are needed for an ISP to be more profitable as an adopter. Notice how the differences decrease close to full technology penetration. The value of $\gamma$ is likely to vary greatly in different countries.

Roaming cost $\left(c_{o}\right.$, Figure 2(d)-2(f)). The relation between a user's entry cost $c_{i}$ and roaming cost $c_{o}$ greatly affects the market diffusion process of user-provided networking. This parameter influences the payoffs of each player. Concerning the ISP, it will determine the interval of technology penetration where the ISP should join the system. A higher $c_{o}$ not only makes the ISP join even at lower penetration, it also assures higher payoffs. On the other hand, the ratio of $c_{i}$ and $c_{o}$ determines the spread of technology among users. If $c_{i}$ is very low it is worth becoming an insider independent of technology penetration.

On the contrary, if $c_{i}$ is relatively high compared to $c_{o}$, users lose the incentive to join. In between, more users are set to turn insiders if there is low or high technology penetration. In case of medium penetration $\left(n_{i} \approx n_{o}\right)$ users experience negative instantaneous payoffs, which may prevent further technology diffusion (see Proposition 1). At last, the most interesting effect surfaces in the context of the mediator: if the roaming cost is significantly higher than the entry cost $\left(c_{o}=30, c_{i}=5\right)$, the maximum payoff is realized at around $65 \%$ technology penetration. One can interpret this phenomenon so as the mediator is not interested in a truly global coverage: she prefers a well-balanced mix of insiders and outsiders.

Putting it to an other perspective, by setting roaming cost $c_{o}$ and entry cost $c_{i}$ properly, the mediator can side either with the users or the ISPs.

Revenue shares ( $\alpha$ and $\beta$, Figure 2(g)-2(i)). It is quite straightforward that a low $\beta$ prevents the ISP from joining at all. What is less than obvious is that a mediator can start business with a higher ISP revenue share $\beta$ and when technology penetration is higher, she can change $\beta$ to a lower value; it will be still beneficial for further ISPs to join, while the remaining surplus can be invested to the user revenue share $\alpha$ or pocketed as profit for the mediator. When this surplus is invested into $\alpha$ it can help the system transition from medium to high penetration (note the negative user payoff in the medium range). Also, it can be observed that in a high penetration case user payoffs with different $\alpha$ converge quickly to each other, meaning that $\alpha$ can be decreased, and the mediator can pocket the 


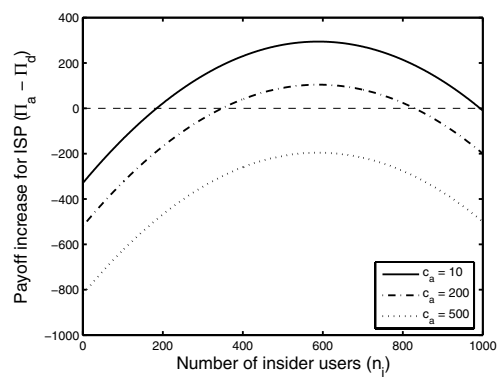

(a) ISP: adoption cost $\left(c_{a}\right)$

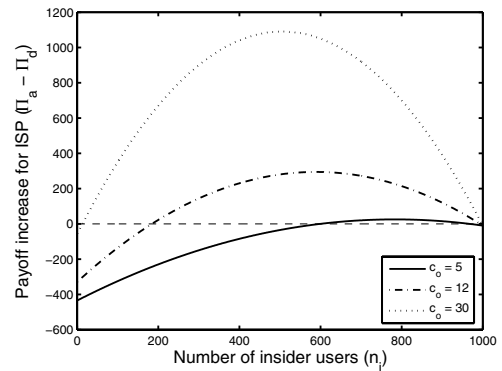

(d) ISP: roaming cost $\left(c_{o}\right)$

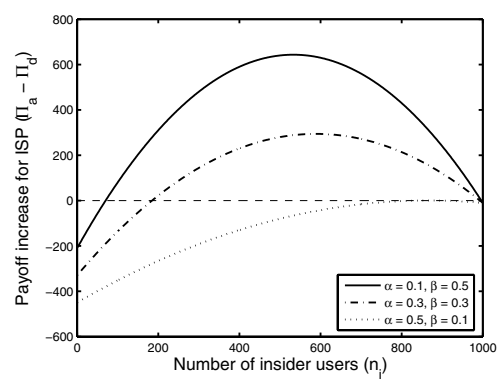

(g) ISP: revenue shares $(\alpha, \beta)$

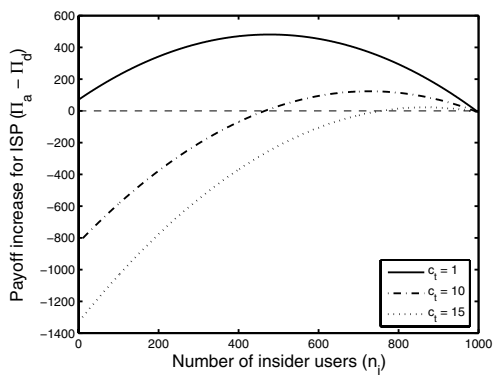

(b) ISP: traffic-dependent cost $\left(c_{t}\right)$

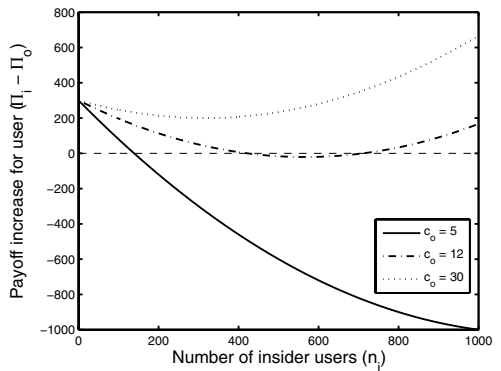

(e) User: roaming cost $\left(c_{o}\right)$

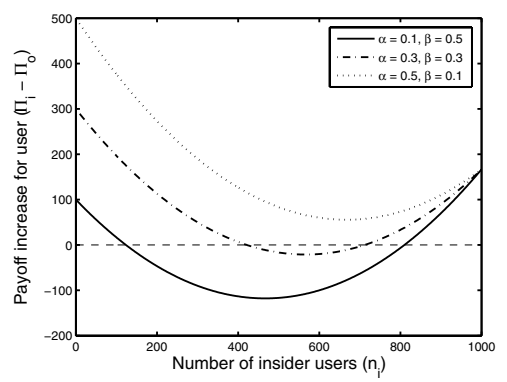

(h) User: revenue shares $(\alpha, \beta)$

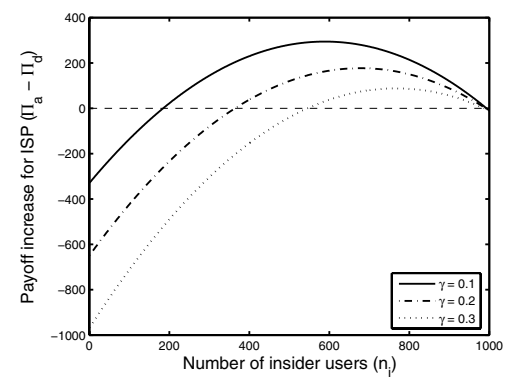

(c) ISP: ratio of risk-taking users $(\gamma)$

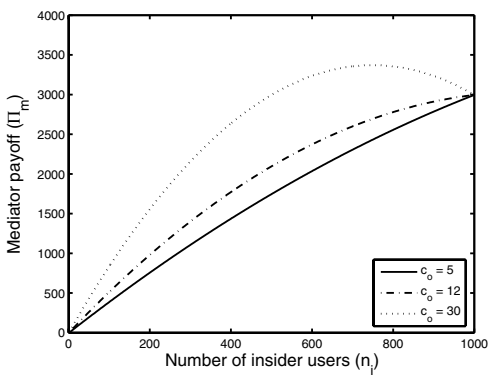

(f) Mediator: roaming cost $\left(c_{o}\right)$

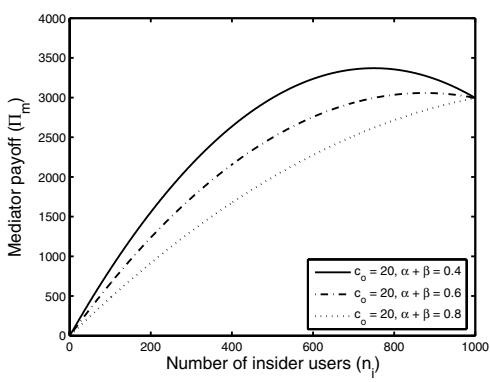

(i) Mediator: revenue shares $(\alpha, \beta)$

Figure 2: Expected revenue for user, ISP and mediator vs. various parameters (default parameter values: $n_{u}=1000, c_{i}=5$, $c_{o}=12, c_{t}=5, c_{a}=10, G=0.5, R=0.001, \alpha=0.3, \beta=0.3, \gamma=0.1$ )

surplus. Furthermore, an increasing share of revenues for the mediator $(1-\alpha-\beta)$ causes her optimal payoff to be realized at lower penetrations.

The mediator can adjust revenue shares over time to shape the technology diffusion process to her own best interest, e.g., going for smooth technology diffusion, maximizing her own profit or maximizing social welfare.

\section{FUTURE WORK}

This study is intended to be a first step towards the understanding of economic interactions in wireless, global, user-provided and ISP-supported networking frameworks. Here we give an outlook at further issues and possible research directions which can bring us closer to a complete picture.

Relaxing strict assumptions. Throughout our analysis we assumed a homogeneous setting, where all users have similar mobility characteristics, demand and utility of being online. We also assumed that insiders are uniformly distributed around the world, and bear the same geographic relevance. While these restrictions enabled us to handle the problem analytically, some (or all) of them can be eliminated by using simulations to evaluate the system in a more diverse user regime.
Furthermore, we only investigated instantaneous costs and utilities in order to compare the payoffs under various parameter settings: this way we analyzed "snapshots" of the system. We intend to use the tools of dynamic and evolutionary game theory to capture the temporal characteristics of the system in an analytic manner, e.g., examining how user participation and technology adoption develops.

Moreover, the assumption of a static user set can be relaxed by introducing a third type of user (a "non-user"), who does not even use shared WiFi. This user may eventually be converted to an outsider or insider, thus dynamically increasing $n_{u}$. Also ISPs may lose subscribers who want to be insiders, but are not willing to violate their broadband contracts. An extended model could also include broadband costs and revenues (both for users and ISPs), and allow for switching providers. Also, insiders could also pay (albeit less) for roaming.

Mediator payoff and business models. While the business model we used in this paper is based on the FON concept, we cannot yet determine if it is optimal in any (social welfare, maximum mediator profit, etc.) aspect. Further research applying mechanism design techniques to the problem of creating and evaluating efficient business models for user-provided networking is highly relevant. 
Additionally, more sophisticated user categories can be introduced to refine revenue flow. On the other hand, the existence of multiple mediators induce competition among them, which can be also taken into consideration. Last, the impact of a global wireless community network on $3 \mathrm{G} / 4 \mathrm{G}$ mobile operators should also be studied (see [8] for guidelines).

Mobility patterns. While a user's mobility pattern greatly influences the decision to opt in, real-world mobility measurements are not easily accessible. The authors of [10] found that humans follow simple reproducible mobility patterns in spite of the diversity of the population. Furthermore, it is becoming known that human mobility is closely related to human relationships, thus the structure of a mobility graph should resemble that of a social network. Such a mobility graph can describe $\mathrm{WiFi}$ access points and their owners as nodes and access sharing relations as directed edges among them, while the frequency of visiting a given location can be reflected by assigning weights to the edges. In such a graph the heavy-tail out-degree distribution, the clustering and small-world effects are intuitively present; hence we believe that a scale-free graph model of complex networks is a promising way to target the user mobility issue.

Traffic measurements. Modeling ISP costs proportional to traffic is far from trivial in this context. For an advanced cost model one should take into account traffic and usage characteristics as measured in the very network. Also, the utility of users is greatly dependent on traffic prioritization techniques and quality of experience. While there are some existing surveys dealing with the FON system [11], we still lack a large-scale measurement study to build upon.

\section{CONCLUSION}

In this paper, we investigated the economic interactions in global wireless community networks. Our key contribution lies in building an analytical model incorporating community mediators, users and ISPs, and showing under what circumstances players benefit from joining the user-provided networking framework. In particular, we revealed the problem of users and ISPs not joining under different technology penetration regimes, and proposed a solution where the community provider dynamically adjusts revenue shares to overcome this issue. Furthermore, we showed how the commu- nity provider can side either with the users or the ISPs by adjusting roaming and entry costs. Our future work will include studying more realistic scenarios, investigating alternative business models from the community provider's view and integrating a more advanced user mobility model into our analysis.

\section{ACKNOWLEDGMENTS}

This work has been supported by HSNLab, Budapest University of Technology and Economics, http: / /www. hsnlab.hu.

\section{REFERENCES}

[1] FON website. http: / / www . fon. com

[2] Whisher website. http://www.whisher.com

[3] WeFi website. http://www. wefi.com

[4] Meraki website. http: / / www .meraki.com

[5] E. C. Efstathiou, P. A. Frangoudis, and G. C. Polyzos. "Stimulating Participation in Wireless Community Networks", In Proceedings of IEEE Infocom 2006, Barcelona, Spain, April 2006.

[6] M. H. Manshaei, J. Freudiger, M. Felegyhazi, P. Marbach, and J.-P. Hubaux. "On Wireless Social Community Networks” In Proceedings of IEEE Infocom 2008, Phoenix, AZ, USA, April 2008.

[7] A. Mazloumian, M. H. Manshaei, M. Felegyhazi, and J.-P. Hubaux. "Optimal Pricing Strategy for Wireless Social Community Networks", In Proceedings of ACM NetEcon 2008, Seattle, WA, USA, August 2008.

[8] A. Zemlianov and G. De Veciana. "Cooperation and decision-making in a wireless multi-provider setting" In Proceedings of IEEE Infocom 2005, Miami, FL, USA, March 2005.

[9] D. Fundenberg and J. Tirole. "Game Theory”, MIT Press, 1991.

[10] M. C. Gonzalez, C. A. Hidalgo, and A.-L. Barabasi. "Understanding individual human mobility patterns", Nature 453, 479-482 (2008).

[11] A. Esenjanovna. "Performance Evaluation of a FON Network", MSc thesis, Asian Institute of Technology, 2007. 